ICRR-Report-514-2004-12

STUPP-05-178

KEK-TH-1013

TUM-HEP-584/05

October 24, 2018

\title{
Significant effects of second KK particles on LKP dark matter physics
}

\author{
Mitsuru Kakizaki ${ }^{a, 1}$, Shigeki Matsumoto ${ }^{a, b, 2}$, Yoshio Sato ${ }^{c, d, 3}$ \\ and Masato Senami ${ }^{a, 4}$ \\ ${ }^{a}$ ICRR, University of Tokyo, Kashiwa 277-8582, Japan \\ ${ }^{b}$ Theory Group, KEK, Oho 1-1, Tsukuba, Ibaraki 305-0801, Japan \\ ${ }^{c}$ Department of Physics, Saitama University, Saitama 338-8570, Japan \\ ${ }^{d}$ Physik-Department, Technische Universität München, \\ James-Franck-Strasse 85748 Garching, Germany
}

\begin{abstract}
We point out that Kaluza-Klein (KK) dark matter physics is drastically affected by second KK particles. In this work various interesting phenomena caused by the second KK modes are discussed. In particular, we reevaluate the annihilation cross section relevant to the thermal relic density of KK dark matter in universal extra dimension models. In these models, the first KK mode of $B$ boson is a viable dark matter candidate by virtue of KK-parity. We demonstrate that the KK dark matter annihilation cross section can be enhanced, compared with the tree level cross section mediated only by first KK particles. The mass of the first KK mode of $B$ boson consistent with the WMAP observation is increased.
\end{abstract}

\footnotetext{
${ }^{1}$ kakizaki@icrr.u-tokyo.ac.jp

${ }^{2}$ smatsu@post.kek.jp

3 yoshio@krishna.th.phy.saitama-u.ac.jp

${ }^{4}$ senami@icrr.u-tokyo.ac.jp
} 


\section{Introduction}

Precise measurements of the cosmological parameters have achieved amazing progress in recent years. Especially observation of cosmic microwave background anisotropies by WMAP revealed that non-baryonic dark matter amounts to 20 percent of the energy of our universe [1. The existence of the dark matter forces us to consider physics beyond the standard model (SM) for its constituent. because there is no candidate for the dark matter in the SM.

On theoretical side weakly interacting massive particles (WIMPs) are considered as excellent candidates for dark matter. One of the excellent candidates is the lightest supersymmetric particle (LSP) present in supersymmetric (SUSY) extensions of the SM. The LSP is stabilized by virtue of R-parity and has been most extensively studied so far [2]. Recently an alternative candidate for WIMP dark matter was proposed in universal extra dimension (UED) models [3], which is a well-motivated scenario invoking TeV-scale extra dimensions [4. In UED models, all SM particles propagate in the compact spatial extra dimensions, leading to the towers of the Kaluza-Klein (KK) partners for each SM particle in four dimensional point of view. The KK mass spectra are quantized and each KK mode is labeled by KK number $n$. Momentum conservation in the compact extra dimension, which is converted to the KK number conservation, guarantees the stability of the lightest KK particle (LKP). The LKP is a viable candidate for dark matter.

The interesting aspect of UEDs is a characteristic feature of the mass spectra. The masses of particles at each KK mode are highly degenerated, because the compactification scale, the inverse of the size $R$ of extra dimensions, should be sufficiently

large in order not to conflict with electroweak precision measurements [5, 6].

In the simplest UED model, the extra dimension is compactified on an $S^{1} / Z_{2}$ orbifold. Orbifolding, required for obtaining chiral zero modes, violates KK number conservation and leaves its remnant called KK-parity. Under the parity particles at even (odd) KK modes have plus (minus) charge. The parity also stabilizes the LKP. This situation is quite similar to the LSP stabilized by R-parity in SUSY models. The first KK mode of $B$ boson, $B^{(1)}$, is found to be the LKP in the minimal setup 7.

There have been several studies on this LKP, which include the calculation of the relic density [8], observational probabilities of dark matter through direct detections [9, 10] and indirect detections 9], 11]-[13, and collider signatures at future accelera- 
tor experiments [14-[16]. In particular its annihilation into fermion pair is discussed extensively because it has potential to account for the positron excess reported by the HEAT experiment [17. It is difficult to explain the anomaly in the context of Majorana dark matter such as LSP.

In preceding studies contributions from first KK modes to various LKP phenomena have been extensively investigated, whereas those from higher KK modes have not been considered. In this paper we emphasize that the effects of second KK particles are important and that it is worth investigating second KK physics in detail. This is because dark matter is non-relativistic from the age of the freeze-out to the present and the incident energy of two LKPs is almost degenerate with the masses of the second KK particles. Thus the LKP pair annihilation processes mediated by second KK particles in the $s$-channel may be significantly enhanced due to the resonances. Such resonances affect the relic abundance and detection probabilities of dark matter in the present universe, and collider phenomenology. The situation should be contrasted with other models such as SUSY models, where tuning of fundamental parameters is required to achieve the resonance enhancement. In this paper we appreciate the 'natural resonance' that incident energy of two first KK modes is close to masses of second KK particles. This feature inherent in UEDs is applicable to many processes in LKP dark matter physics.

In particular we reexamine LKP abundance as one of the most important examples in the second KK particle physics based on the minimal model. We find that the $s$-channel dark matter annihilation process mediated by the second KK mode of the neutral scalar Higgs boson, $h^{(2)}$, competes with the tree level processes in which no second KK particles contribute. As a result the LKP mass consistent with the WMAP observation is increased compared with that indicated in the preceding work [8].

The outline of this paper is as follows: in Sec. 2 we briefly review the minimal UED model based on an $S^{1} / Z_{2}$ orbifold. In Sec. 3 we evaluate LKP dark matter annihilation cross section. We find the enhancement of the cross section due to the resonance by the second KK Higgs boson $h^{(2)}$. In Sec. 4 the relic abundance of the LKP dark matter is calculated. The relic density of LKP turns out to be reduced by the enhanced cross section. Other interesting phenomenology caused by the second KK particles will be discussed in Sec. 5. Section 6 contains our conclusions. 


\section{Universal extra dimension model}

We will briefly review the universal extra dimension model, which is based on the hypothesis that all SM particles propagate in the compact spatial extra dimensions beyond the usual three dimensional space. One of the attractive features of UEDs is to provide a good candidate for cold dark matter.

At first we present the setup used in this letter. Following the minimal UED model, we postulate the field contents to be same as those of the SM. We have three gauge fields $G, W$ and $B$, and one Higgs doublet $H$. The matter contents are three generations of fermions: the quark doublets $Q$, the up- and down-type quark singlets $U$ and $D$, the lepton doublets $L$ and the charged lepton singlet $E$. The extra dimension is compactified by an $S^{1} / Z_{2}$ orbifold. Under the $Z_{2}$ parity we assign plus charge to the left-handed doublets and the right-handed singlets so that their zero modes coincide with the SM chiral fermions. The Higgs doublet must carry plus charge in order to allow the usual Yukawa couplings.

In the four dimensional perspective the zero modes are identified with the SM particles and their interactions are same as those in the SM. The electroweak symmetry breaking also mixes the hypercharge gauge boson $B$ and the neutral $\mathrm{SU}(2)_{L}$ gauge boson $W^{3}$ at each KK level. Since the mass difference between $B^{(n)}$ and $W^{(n)}$ $(n \geq 1)$ induced from radiative corrections is larger than the electroweak scale, we can neglect their mixing angles. Therefore $B^{(n)}$ and $W^{(n)}$ are regarded as the mass eigenstates.

There are some possible candidates for LKP dark matter, which must be neutral: first KK states of the neutral gauge bosons, neutral Higgs bosons and neutrinos. The $W$ boson, the Higgs boson and the lepton doublet carry $\mathrm{SU}(2)_{L}$ quantum number

while the $B$ boson is singlet under the SM gauge group. Thus radiative corrections increase the masses of $W^{(1)}, H^{(1)}$ and $L^{(1)}$, leaving the mass of $B^{(1)}$ almost unchanged [7. As a result, $B^{(1)}$ is the LKP.

\section{Annihilation cross section for LKP dark matter}

In this section we evaluate annihilation cross section for LKP dark matter. We focus on resonant $\mathrm{KK}$ dark matter annihilation process into zero mode (SM) particles, which proceeds with $s$-chanel $h^{(2)}$ as illustrated in Fig. 1, The $s$-chanel annihilation at tree level into two first KK particles and those into a second KK particle and an 


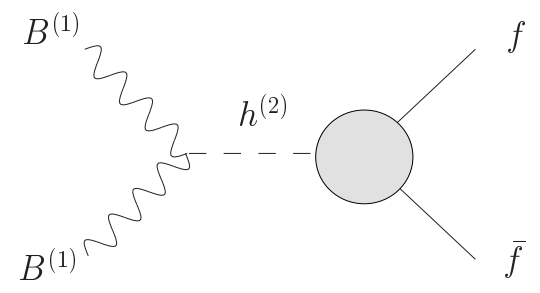

Figure 1: The resonant $B^{(1)}$ annihilation process mediated by $s$-channel $h^{(2)}$ into a zero mode (SM) particle-anti-particle pair $f \bar{f}$.
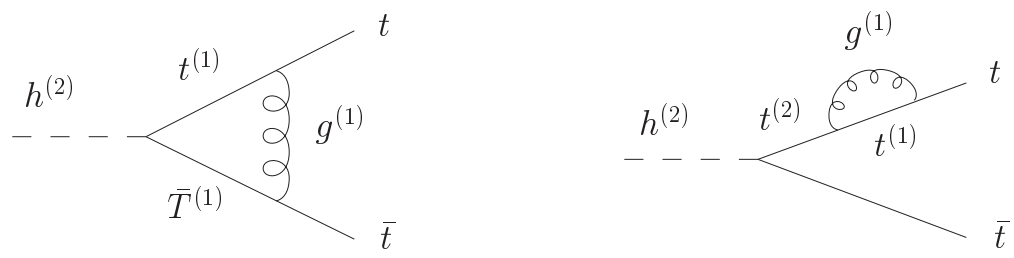

Figure 2: The dominant one-loop diagrams leading to the resonant $B^{(1)}$ pair annihilation process mediated by $h^{(2)}$. Here $t$ is the (zero-mode) top quark, and $t^{(1)}, T^{(1)}$ and $g^{(1)}$ represent the first KK modes of left- and right-handed top quarks and gluon respectively.

SM one are kinematically forbidden or extremely suppressed by their small Yukawa couplings. On the other hand, interactions among one second KK particle and two SM particles are necessarily generated through radiative corrections and thus dominantly contribute to annihilation processes. We survey possible diagrams leading to such resonant annihilations and find that the dominant contribution comes from the one-loop diagrams depicted in Fig. 2.

We have calculated the annihilation cross section including the effect of the $h^{(2)}$ resonance: $\sigma=\sigma_{\text {res }}+\sigma_{\text {tree }}$. The first term $\sigma_{\text {res }}$ arises from the resonant one-loop diagrams as

$$
\sigma_{\mathrm{res}}=\frac{\pi \alpha_{\mathrm{em}} \tan ^{2} \theta_{W} m_{Z}^{2}}{9 m \beta} \frac{\Gamma_{h^{(2)}}}{\left(s-m_{h^{(2)}}^{2}\right)^{2}+m_{h^{(2)}}^{2} \Gamma_{h^{(2)}}^{2}}\left(3+\frac{s\left(s-4 m^{2}\right)}{4 m^{4}}\right),
$$

where $\alpha_{\mathrm{em}}$ and $\theta_{W}$ represent the fine-structure constant and weak-mixing angle, $m$ and $m_{h^{(2)}}$ are the masses of $B^{(1)}$ and $h^{(2)}$ respectively, $s$ is the center-of-mass energy squared and $\beta^{2}=1-4 \mathrm{~m}^{2} / \mathrm{s}$. The decay rate of $h^{(2)}$, dominated by the mode into the $t \bar{t}$ pair as discussed above, is written

$$
\Gamma_{h^{(2)}}=\frac{y_{t}^{2} \alpha_{s}^{2} m_{h^{(2)}}}{384 \pi^{3}}\left[\ln \left(\frac{\Lambda^{2}}{\mu^{2}}\right)\right]^{2}
$$


where $\alpha_{s}$ and $y_{t}$ are the strong and top Yukawa coupling constants respectively. In the logarithm, $\Lambda$ denotes the cutoff scale of the theory and $\mu$ represents the renormalization scale characterizing the typical energy, which is set to be $\mu=m(\simeq$ $R^{-1}$ ). Here, we take the leading logarithmically divergent part into account in the calculation of the cross section. Resonant dark matter annihilation is naturally realized in the framework of UEDs because $s \simeq(2 m)^{2} \simeq m_{h^{(2)}}^{2}$. On the other hand, the last term $\sigma_{\text {tree }}$ stems from the tree level diagrams, in which only first KK particles are exchanged [8]:

$$
\sigma_{\text {tree }}=\frac{95 \pi \alpha_{\mathrm{em}}^{2}}{81 \cos ^{4} \theta_{W}} \frac{10\left(2 m^{2}+s\right) \tanh ^{-1} \beta-7 s \beta}{s^{2} \beta^{2}}+\frac{\pi \alpha_{\mathrm{em}}^{2}}{6 \cos ^{4} \theta_{W} s \beta} .
$$

The interferential contribution between the tree-level diagram into $t \bar{t}$ and the oneloop diagrams is negligible because it suffers from the chirality suppression of the top quark mass.

We have numerically calculated the thermally averaged annihilation cross section (multiplied by the relative velocity between incident $B^{(1)}$ bosons: $v=\sqrt{s} \beta / m$ ) in the non-relativistic limit, which is given by

$$
\langle\sigma v\rangle=4 \pi\left(\frac{m}{4 \pi T}\right)^{3 / 2} \int_{0}^{\infty} d v v^{2} e^{-m v^{2} / 4 T} \sigma v,
$$

at temperature $T$. It is important to evaluate the thermal average of the cross section at the freeze-out temperature because it affects the calculation of the abundance of the thermal relic. In case of the UED model the LKP decouples from thermal equilibrium at $T \sim m / 25$. Figure B(a) shows the contour plots of the predicted annihilation cross section in units of $10^{-26} \mathrm{~cm}^{3} \mathrm{sec}^{-1}$. The ratio of the total cross section to that at tree level is also depicted in Fig. [3(b). We choose the temperature as $T=m / 25$. The cutoff scale is set to be $\Lambda R=20$, which is a characteristic value assumed in the UED scenarios. Here we treat $m_{h^{(2)}}$ as a free parameter instead of the Higgs mass and show the dependence of the cross sections on the mass splitting $\delta \equiv\left(m_{h^{(2)}}-2 m\right) / 2 m$. These figures demonstrate that the resonant process competes with those at tree level, resulting in increased annihilation cross section. For $\delta \sim 1 \%$, the incident energy of the LKP pair matches the $h^{(2)}$ pole and leads to enhancement. It is interesting to notice that $\delta \simeq 1 \%$ is indeed realized in a wide region of the parameter space in the minimal UED [7]. 


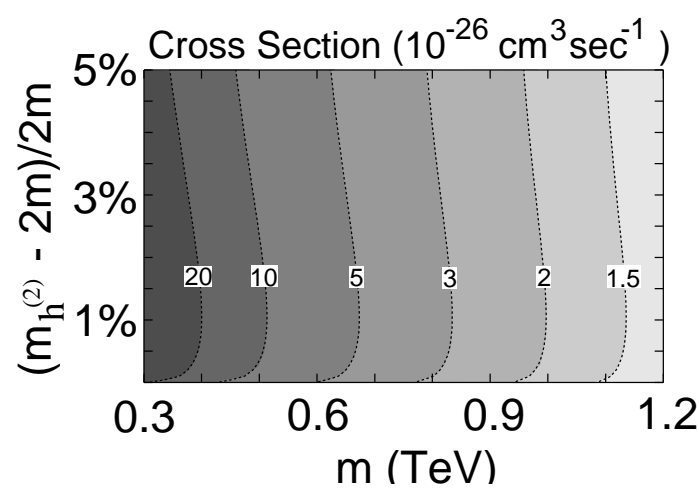

(a)

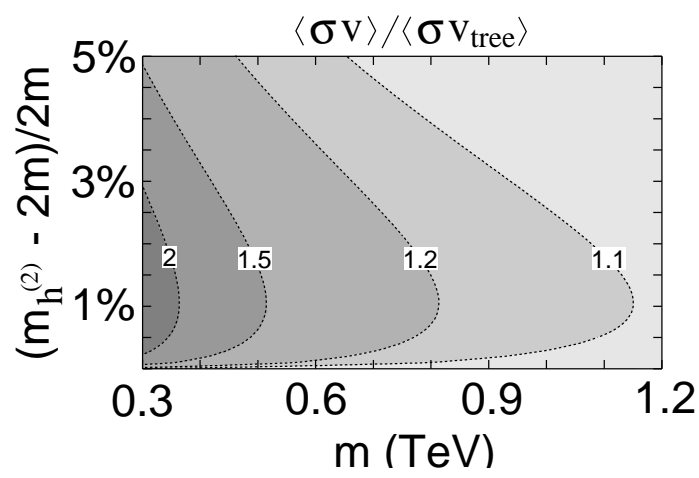

(b)

Figure 3: (a) Contour plot of the averaged annihilation cross section (multiplied by the relative velocity) for the LKP, $B^{(1)}$. (b) Contour plot of the ratio of the total averaged cross section to the tree level one. The masses of $B^{(1)}$ and $h^{(2)}$ are represented by $m$ and $m_{h}^{(2)}$ respectively. Here we set the temperature to be $T=m / 25$.

\section{Thermal relic density of LKP dark matter}

We now calculate the thermal relic density of the LKP dark matter using annihilation cross section obtained in Sec. 3. The time evolution of the LKP number density $n$ obeys the following Boltzmann equation:

$$
\frac{d n}{d t}+3 H n=-\langle\sigma v\rangle\left(n^{2}-n_{\mathrm{eq}}^{2}\right)
$$

where $H$ is the Hubble parameter describing the expansion of the universe. In the non-relativistic limit the equilibrium number density $n_{\mathrm{eq}}$ at temperature $T$ is given by

$$
n_{\mathrm{eq}}=g\left(\frac{m T}{2 \pi}\right)^{3 / 2} e^{-m / T},
$$

where the number of degree of freedom is $g=3$ for the LKP. Due to the conservation of entropy per comoving volume, the Boltzmann equation is rewritten

$$
\frac{d Y}{d x}=-\frac{\langle\sigma v\rangle}{H x} s\left(Y^{2}-Y_{\mathrm{eq}}^{2}\right)
$$

where $Y=n / s$ and $Y_{\text {eq }}=n_{\text {eq }} / s$ and $x=m / T$ with entropy $s=2 \pi^{2} g_{*} T^{3} / 45$. Here $g_{*}$ denotes the number of relativistic degrees of freedom. By solving the Boltzmann equation we obtain the present number density over entropy of dark matter $Y_{\infty}$. It is useful to express the relic density in terms of $\Omega h^{2}$. Here $\Omega$ is the ratio of the dark matter energy density to the critical density in the present universe. The dark 


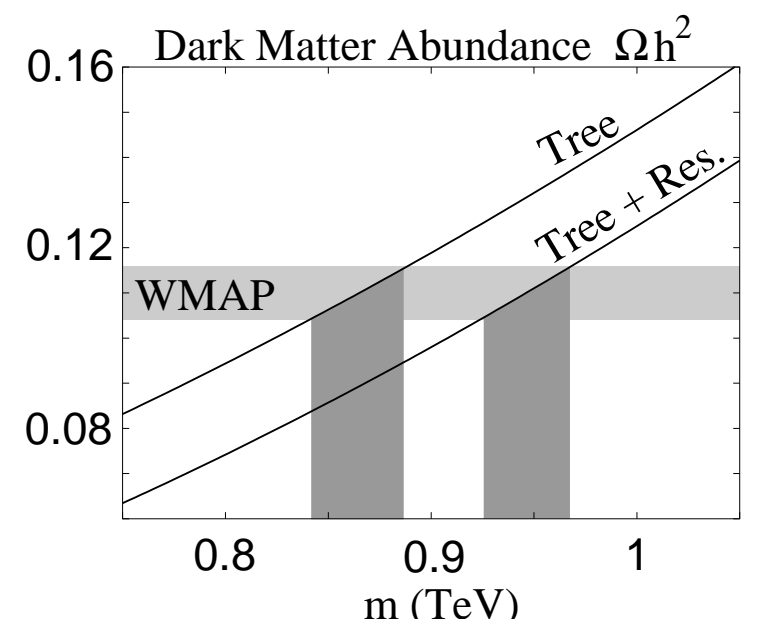

Figure 4: The predicted dark matter abundance $\Omega h^{2}$ as a function of the LKP mass $m$ including resonance (Tree + Res.) for $g_{*}=100$. Here we set the cutoff scale to be $\Lambda R=20$ and the mass splitting $\delta=1 \%$. For comparison, we show the tree level result (Tree). The $1 \sigma$ region of the relic abundance measured by WMAP is also shown: $\Omega h^{2}=0.110 \pm 0.006$. The allowed mass regions are highlightened in both cases.

matter density is given by $\rho=m n=m s_{0} Y_{\infty}$, with $s_{0}$ being the present entropy $s_{0}=2900 \mathrm{~cm}^{-3}$. The critical density is $\rho_{c}=3 H_{0}^{2} M_{\mathrm{Pl}}^{2} / 8 \pi=1.1 \times 10^{-5} h^{2} \mathrm{GeV} \mathrm{cm}^{-3}$, where the Planck mass is $M_{\mathrm{Pl}}=1.2 \times 10^{19} \mathrm{GeV}$ and $H_{0}$ is the present Hubble expansion rate parameterized as $H_{0}=100 h \mathrm{~km} \mathrm{sec}^{-1} \mathrm{Mpc}^{-1}$. The WMAP data is fitted by $h=0.72 \pm 0.05[1]$.

We numerically solve the Boltzmann equation in Eq. (17) to obtain the thermal relic density. Figure 4 shows the predicted dark matter abundance $\Omega h^{2}$ (Tree + Res.) as a function of the LKP mass $m$ for $g_{*}=100$. Here we take $\Lambda R=20, \delta=1 \%$ for UED parameters. For comparison, we show the tree-level result (Tree) [8]. The $1 \sigma$ region of the relic abundance measured by WMAP is also shown: $\Omega h^{2}=0.110 \pm$ 0.006. The mass regions accounting for the dark matter density are highlightened in both cases. As a consequence of the resonant annihilation, the mass of the KK dark matter consistent with the WMAP data turns out to be around $950 \mathrm{GeV}$, which is $\sim 100 \mathrm{GeV}$ above the tree-level result. The relic abundance depends on the mass splitting $\delta$, although the numerical difference is small: the allowed region is found to be $m \simeq 900-1000 \mathrm{GeV}$ for $0.5 \%<\delta<2 \%$. We have also checked that the relic abundance is almost insensitive to the cutoff scale $\Lambda$. We conclude that the reduction of the LKP dark matter abundance due to the second KK resonance is a 


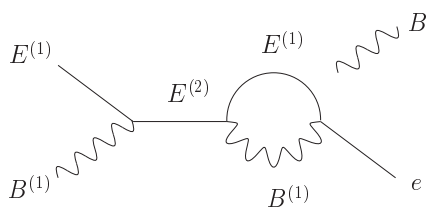

(a)

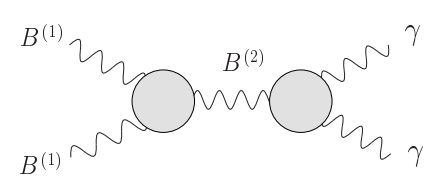

(b)

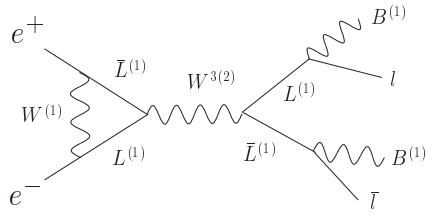

(c)

Figure 5: Resonant processes induced by second KK particles which influence (a) coannihilation, (b) indirect detection and (c) collider phenomenology. In the diagrams $e, l$ and $\gamma$ denote the zero mode (SM) electron, charged lepton and photon, $E^{(1)}, L^{(1)}$ and $B^{(1)}$ represent the first KK modes of right- and left-handed charged lepton, $B$ and Higgs boson, and $E^{(2)}, B^{(2)}$ and $W^{(2)}$ stand for the second KK modes of the right-handed charged lepton, $B$ and $W$ bosons.

characteristic feature of UED models and independent of the detailed mass spectra.

\section{$5 \quad$ Phenomena affected by second KK particles}

In Sec. 团we observed that the predicted relic density of LKP dark matter is significantly changed by the resonance caused by the intermediate second KK Higgs boson compared with the tree level result. Here we discuss other phenomenological issues originating from second KK physics. The 'natural resonance' is a generic feature appearing in UEDs and applicable to various processes of LKP dark matter physics. In the following we consider the cases where the second KK physics plays an essential role in making exciting predictions:

Coannihilation: The predicted relic density of dark matter is drastically changed by the so-called coannihilation when other particles are highly degenerate with the LKP in mass. For the tree level calculation of the relic density, it is known that the LKP mass consistent with WMAP decreases in the presence of the degenerate first KK right-handed electron $E^{(1)}[8]$. The self-annihilation rates of $B^{(1)}$ and $E^{(1)}$ are of the same order while the coannihilation rate is rather suppressed. Then after decoupling from thermal equilibrium, more relics are left over, which lowers the allowed LKP mass. However the coannihilation of $B^{(1)}$ with $E^{(1)}$ is also affected by 'natural resonance' through $s$-channel $E^{(2)}$ exchange accompanied by dipole-type

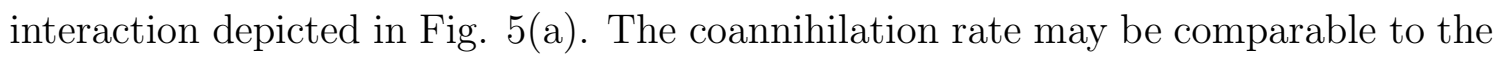
self-annihilation rates under certain circumstances, and the allowed LKP mass is also modified from that calculated at tree level by coannihilation. The detailed analysis including the coannihilation effects will be presented in the subsequent paper [18. 
Indirect detection: Since dark matter is almost at rest in the present universe, the energy of two LKPs matches masses of second KK modes. Especially the relation $m_{B^{(2)}}=2 m_{B^{(1)}}$ is almost retained even after inclusion of radiative corrections to mass spectra. Thus, large annihilation cross sections are induced by the two-loop processes mediated by $B^{(2)}$ in the $s$-channel as depicted in Fig 5 (b). The monochromatic gamma-rays are produced by the LKP annihilations [12]. We find that the resonant $B^{(2)}$ process into two photons at the two-loop level gives a contribution comparable to the one-loop processes mediated by $(\mathrm{KK})$ fermions. The LKP annihilations also directly yield a large number of positrons whose energy is equal to the LKP mass, in contrast to the LSP case. The contribution from the $B^{(2)}$ resonance is found to change the predicted positron flux by $\mathcal{O}(10)$ percent. We leave these interesting subjects to future works.

Collider signatures: Future accelerator experiments are very promising to probe UEDs. In the simple $S^{1} / Z_{2}$ case the discovery reach at the Large Hadron Collider will extend to $R^{-1} \sim 1.5 \mathrm{TeV}$ [15], well above the compactification scale favored by the LKP dark matter scenario. Furthermore, lepton colliders with sufficient centerof-mass energy could conclusively test the UED model due to the 'natural resonance'. Let us consider pair production of the first KK left-handed charged lepton mediated by the $s$-channel second KK mode of $W$ boson as shown in Fig. 5(c). Each product decays into a charged lepton and the LKP carrying large missing energy. The signal event of two charged leptons plus missing energy is expected with significantly large cross section and almost background-free [19]. Since the degeneracy between the intermediate particle mass and twice the LKP mass is a characteristic feature in the framework of UEDs, we could discriminate UEDs from other new physics.

\section{Conclusion}

We have investigated the effects from the second KK particles on low energy predictions. We especially focused on the relic abundance of LKP dark matter, which is the first $\mathrm{KK}$ mode of $B$ boson, $B^{(1)}$. We have pointed out that due to the $h^{(2)}$ resonance the dark matter annihilation cross section is enhanced compared to that involving only the first KK modes. As a result the allowed value of $B^{(1)}$ mass constrained by the WMAP observation is shifted to a larger mass region. We have also discussed various interesting phenomena caused by the second KK modes including coannihilation, indirect detection and collider signatures. Studies involving 'natural 
resonance' will make tremendous advance in KK particle physics.

\section{Acknowledgments}

The work of M.K. is supported in part by the Japan Society for the Promotion of Science.

\section{References}

[1] D. N. Spergel et al. [WMAP Collaboration], Astrophys. J. Suppl. 148 (2003) 175; C. L. Bennett et al., Astrophys. J. Suppl. 148 (2003) 1.

[2] For reviews, see for instance, G. Jungman, M. Kamionkowski and K. Griest, Phys. Rept. 267 (1996) 195; L. Bergstrom, Rept. Prog. Phys. 63 (2000) 793; G. Bertone, D. Hooper and J. Silk, Phys. Rept. 405 (2005) 279; C. Munoz, Int. J. Mod. Phys. A 19 (2004) 3093.

[3] E. W. Kolb and R. Slansky, Phys. Lett. B 135 (1984) 378; K. R. Dienes, E. Dudas and T. Gherghetta, Nucl. Phys. B 537 (1999) 47.

[4] I. Antoniadis, Phys. Lett. B 246 (1990) 377; N. Arkani-Hamed, S. Dimopoulos and G. R. Dvali, Phys. Lett. B 429 (1998) 263.

[5] T. Appelquist, H. C. Cheng and B. A. Dobrescu, Phys. Rev. D 64 (2001) 035002.

[6] K. Agashe, N. G. Deshpande and G. H. Wu, Phys. Lett. B 511 (2001) 85; K. Agashe, N. G. Deshpande and G. H. Wu, Phys. Lett. B 514 (2001) 309; T. Appelquist and B. A. Dobrescu, Phys. Lett. B 516 (2001) 85; F. J. Petriello, JHEP 0205 (2002) 003; T. Appelquist and H. U. Yee, Phys. Rev. D 67 (2003) 055002; D. Chakraverty, K. Huitu and A. Kundu, Phys. Lett. B 558 (2003) 173; A. J. Buras, M. Spranger and A. Weiler, Nucl. Phys. B 660 (2003) 225; J. F. Oliver, J. Papavassiliou and A. Santamaria, Phys. Rev. D 67 (2003) 056002; A. J. Buras, A. Poschenrieder, M. Spranger and A. Weiler, Nucl. Phys. B 678 (2004) 455.

[7] H. C. Cheng, K. T. Matchev and M. Schmaltz, Phys. Rev. D 66 (2002) 036005.

[8] G. Servant and T. M. P. Tait, Nucl. Phys. B 650 (2003) 391.

[9] H. C. Cheng, J. L. Feng and K. T. Matchev, Phys. Rev. Lett. 89 (2002) 211301. 
[10] G. Servant and T. M. P. Tait, New J. Phys. 4 (2002) 99; D. Majumdar, Phys. Rev. D 67 (2003) 095010.

[11] D. Hooper and G. D. Kribs, Phys. Rev. D 67 (2003) 055003; G. Bertone, G. Servant and G. Sigl, Phys. Rev. D 68 (2003) 044008; L. Bergstrom, T. Bringmann, M. Eriksson and M. Gustafsson, arXiv:astro-ph/0410359 E. A. Baltz and D. Hooper, arXiv:hep-ph/0411053

[12] L. Bergstrom, T. Bringmann, M. Eriksson and M. Gustafsson, arXiv:hep-ph/0412001.

[13] D. Hooper and G. D. Kribs, Phys. Rev. D 70 (2004) 115004; D. Hooper and J. Silk, arXiv:hep-ph/0409104.

[14] T. G. Rizzo, Phys. Rev. D 64 (2001) 095010.

[15] H. C. Cheng, K. T. Matchev and M. Schmaltz, Phys. Rev. D 66 (2002) 056006.

[16] C. Macesanu, C. D. McMullen and S. Nandi, Phys. Rev. D 66 (2002) 015009; C. Macesanu, C. D. McMullen and S. Nandi, Phys. Lett. B 546 (2002) 253; G. Bhattacharyya, P. Dey, A. Kundu and A. Raychaudhuri, arXiv:hep-ph/0502031.

[17] S. W. Barwick et al. [HEAT Collaboration], Astrophys. J. 482 (1997) L191;

S. Coutu et al., Astropart. Phys. 11 (1999) 429; J. J. Beatty et al., Phys. Rev. Lett. 93 (2004) 241102.

[18] M. Kakizaki, S. Matsumoto, Y. Sato and M. Senami, in preparation.

[19] M. Battaglia, A. Datta, A. De Roeck, K. Kong and K. T. Matchev, arXiv:hep-ph/0502041. After completion of our work, we received this preprint which deals with collider signatures induced by the $s$-channel resonances. Their work is a good example indicating the importance of second KK particles. 